\title{
Managing Adverse Events Associated with Dinutuximab Beta Treatment in Patients with High-Risk Neuroblastoma: Practical Guidance
}

\author{
Giuseppe Barone ${ }^{1} \cdot$ Ailish Barry $^{1} \cdot$ Francisco Bautista $^{2} \cdot$ Bénédicte Brichard $^{3} \cdot$ Anne-Sophie Defachelles $^{4}$.

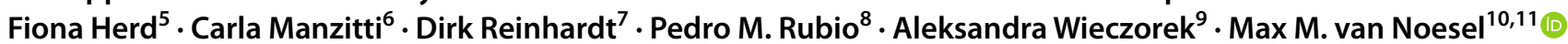

Accepted: 20 August 2021 / Published online: 20 September 2021

(C) The Author(s) 2021

\begin{abstract}
Neuroblastoma is the most common extracranial solid tumour in children, accounting for $15 \%$ of all paediatric cancer deaths. High-risk neuroblastoma is a particularly challenging-to-treat form of disease that requires multimodality treatment, consisting of chemotherapy, surgery, high-dose chemotherapy with autologous haematopoietic stem cell rescue, radiotherapy and differentiation therapy. However, despite intense multimodal treatment regimens, the prognosis for this patient population remains poor. In recent years, immunotherapy with anti-disialoganglioside 2 (anti-GD2) antibodies was found to improve survival rates for patients with high-risk neuroblastoma. Based on studies led by the SIOPEN (International Society of Paediatric Oncology European Neuroblastoma) group, the anti-GD2 antibody dinutuximab beta was approved for use in high-risk neuroblastoma by the European Medicines Agency and has been implemented into the standard of care in many countries across Europe. However, immunotherapy with dinutuximab beta is associated with a number of adverse events that may be challenging for clinicians, such as pain, fever, hypersensitivity reactions and capillary leak syndrome. While these adverse events are considered manageable, there are currently no formal guidelines to support clinicians with their management. The aim of this article is to discuss the management of the most common adverse events encountered in clinical practice and to provide practical guidance to assist clinicians in minimising toxicity associated with dinutuximab beta.
\end{abstract}

\section{Key Points}

Dinutuximab beta can cause adverse events that warrant consideration and management.

Most common adverse events include pain, fever, allergy and capillary leak syndrome.

Practical guidance is provided on the management of the most common adverse events.

Max M. van Noesel

M.M.vanNoesel@prinsesmaximacentrum.nl

Extended author information available on the last page of the article

\section{Introduction}

Neuroblastoma (NB) is an aggressive malignant tumour of the peripheral nervous system that predominantly occurs in children aged $<5$ years and accounts for $15 \%$ of all paediatric deaths due to cancer $[1,2]$. Because of its clinical heterogeneity, the treatment of NB is risk based [3]. Risk is stratified into low, intermediate and high, based on specific clinical and biological features, such as age at time of diagnosis (18 months cut-off), tumour histology and molecular profile (MYCN amplification, segmental chromosomal aberrations and ploidy) [3]. Children with high-risk NB account for 50\% of cases and have poor prognosis (survival rates $<50 \%$ ) despite multimodality treatment (induction chemotherapy, surgery, high-dose chemotherapy with autologous haematopoietic stem cell transplant, radiotherapy and differentiation therapy) [4, 5]. Anti-disialoganglioside 2 (antiGD2)-based immunotherapy has changed the outlook for patients with high-risk NB [1]. The addition of the anti-GD2 antibody dinutuximab (Unituxin ${ }^{\circledR}$ ) to the standard of care 
has improved outcomes (survival rates $>60 \%$ ) in patients with high-risk NB in the COG-ANBL0032 study, which led to the approval of this antibody in the USA [6-8]. A similar anti-GD2 antibody, dinutuximab beta (Qarziba $\left.{ }^{\circledR}\right)$, has been added to the current standard of care for patients with high-risk NB in Europe (Fig. 1a), based on the positive results of the SIOPEN (International Society of Paediatric Oncology European Neuroblastoma) studies [9-11]. However, treatment with these chimeric antibodies is associated with a number of adverse events (AEs) that warrant special consideration and management. Although the incidence and severity of AEs have been widely reported, little guidance is available in the literature regarding the management of common AEs in children receiving dinutuximab beta. Training clinicians and nurses on how to properly manage these AEs is important to ensure safe administration of immunotherapy and to avoid unplanned dose and schedule modifications that may result in reduced efficacy.

This review provides a brief overview of the toxicity profile associated with different treatment schedules of dinutuximab beta in children with NB and aims to provide clinical guidance for the management of the most frequently encountered AEs to support clinical decision-making by healthcare professionals.

\section{Toxicity Profiles Associated with Different Treatment Schedules of Dinutuximab Beta}

Prior to the development of dinutuximab beta, a similar antiGD2 antibody called dinutuximab was developed and investigated by the Children's Oncology Group (COG) $[6,7,13]$. While the development of dinutuximab and dinutuximab beta was based on the same anti-GD2 antibody construct (ch14.18), they were produced in different cell lines (dinutuximab in mouse SP2/0 cells; dinutuximab beta in Chinese hamster ovary [CHO] cells), resulting in different posttranslational glycosylation patterns $[8,9,13]$. Dinutuximab was approved for use by the Food and Drug Administration (FDA) based on the COG trial ANBL0032, which showed that dinutuximab combined with cytokines (granulocyte macrophage colony-stimulating factor and interleukin-2 [IL-2]) and isotretinoin prolonged event-free survival and a

1. Induction therapy

Induction

chemotherapy
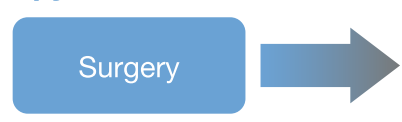

2. Consolidation therapy

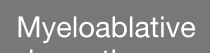

chemotherapy

Stem cell

transplant
3. Maintenance therapy

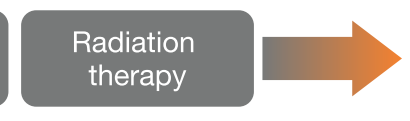

Immunotherapy with dinutuximab beta b Short-term infusion schedule

Dinutuximab beta $20 \mathrm{mg} / \mathrm{m}^{2} /$ day

over 8 hours for 5 days

(total dose: $100 \mathrm{mg} / \mathrm{m}^{2}$ )

IL-2 $6 \times 10^{6} \mathrm{IU} / \mathrm{m}^{2} /$ day

Isotretinoin (160 mg/m²/day bid)

\section{Long-term infusion schedule}

Dinutuximab beta $10 \mathrm{mg} / \mathrm{m}^{2} /$ day continuous infusion over 10 days (total dose: $100 \mathrm{mg} / \mathrm{m}^{2}$ )

IL-2 $3 \times 10^{6} \mathrm{IU} / \mathrm{m}^{2} /$ day

Isotretinoin $\left(160 \mathrm{mg} / \mathrm{m}^{2} /\right.$ day bid)

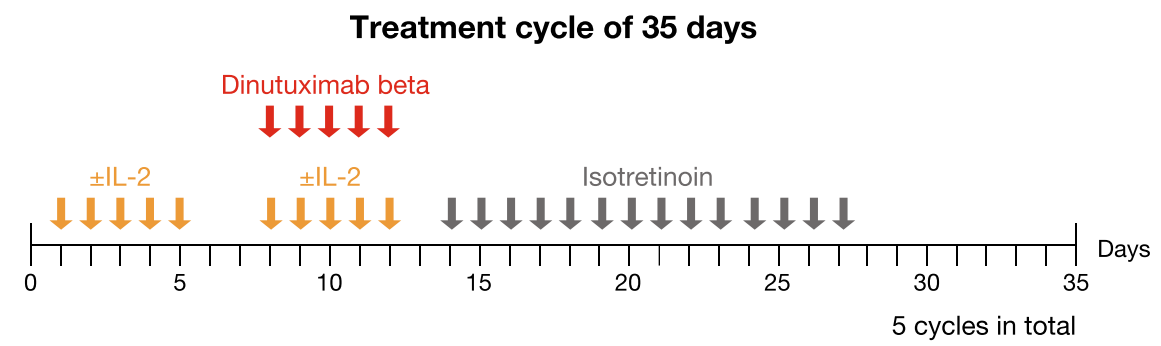

Treatment cycle of 35 days

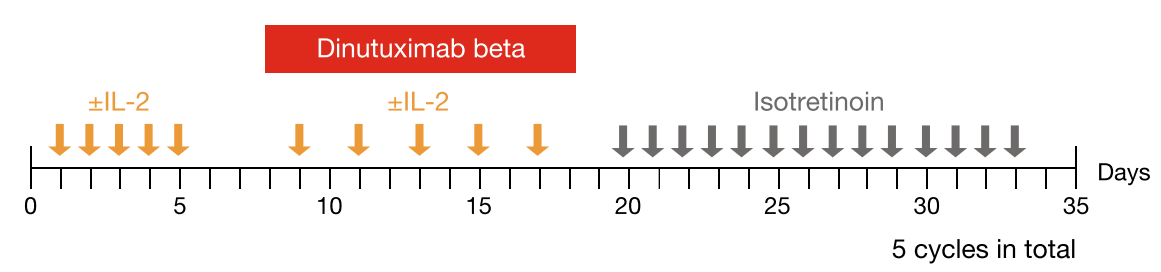

Fig. 1 a SIOPEN treatment pathway in high-risk neuroblastoma. b Short-term and long-term infusion treatment schedules of dinutuximab beta for the treatment of patients with high-risk neuroblastoma

in the SIOPEN studies [5, 10, 11]. Bid twice daily, IL-2 interleukin-2, SIOPEN International Society of Paediatric Oncology European Neuroblastoma 
overall survival in patients with high-risk NB compared with isotretinoin alone [6-8].

Maintenance therapy with dinutuximab beta was investigated as part of a long-term study undertaken by the SIOPEN group to improve outcomes of children with high-risk NB treated according to the European protocol $[10,11,14]$. The SIOPEN group tested two different treatment schedules of dinutuximab beta (Fig. 1b): short-term infusion (STI) and long-term infusion (LTI) $[10,11]$. The initial STI study investigated whether the addition of IL-2 to dinutuximab beta STI $\left(100 \mathrm{mg} / \mathrm{m}^{2}\right.$ given over $8 \mathrm{~h}$ for 5 days) could increase survival in patients with high-risk NB who responded to standard SIOPEN induction and consolidation therapy [10]. However, adding IL-2 to dinutuximab beta STI and isotretinoin did not improve patient outcomes, but substantially increased toxicity compared with dinutuximab beta and isotretinoin alone [10]. Because clinical observations suggested that immunotherapy-related pain and other side effects could be reduced by decreasing the infusion rate [12], the SIOPEN group next investigated whether dinutuximab beta given as LTI $\left(100 \mathrm{mg} / \mathrm{m}^{2}\right.$ given over 10 days as continuous infusion) was associated with a better toxicity profile than the STI schedule [11]. Similar to the STI study, the potential of IL-2 to improve patient outcomes was also assessed in the LTI study. To reduce AEs, IL-2 was administered at half the dose used in the STI study (Fig. 1b). The addition of IL-2 to dinutuximab beta LTI plus isotretinoin substantially increased toxicity, without improving survival in children with high-risk NB [11]. The most common grade 3/4 AEs (according to the National Cancer Institute Common Toxicity Criteria [NCI-CTCAE]) reported in the SIOPEN studies included pain, fever, hypersensitivity reactions/allergy, capillary leak syndrome, diarrhoea and elevated liver enzymes (Table 1) $[10,11]$. While all of these AEs were experienced by patients receiving either treatment schedule, the incidence of immunotherapy-related pain, hypersensitivity reactions and hypotension was lower with the LTI compared with the STI regimen in patients receiving dinutuximab beta alone (Table 1) [10,11]. Therefore, not only the omission of IL-2 but also the use of the LTI schedule were associated with an improved toxicity profile. Based on these findings, SIOPEN recommends dinutuximab beta LTI without IL-2 as the standard of care for patients with high-risk NB receiving immunotherapy [11]. Since this treatment regimen is now predominantly used in clinical

Table 1 Selected grade 3 and 4 adverse events reported with dinutuximab beta short-term infusion (100 mg/m $\mathrm{m}^{2}$ given over $8 \mathrm{~h}$ for 5 days $)$ and long-term infusion $\left(100 \mathrm{mg} / \mathrm{m}^{2}\right.$ given over 10 days) in patients with high-risk neuroblastoma in the SIOPEN studies [11]

\begin{tabular}{|c|c|c|c|c|}
\hline \multirow[t]{2}{*}{ Selected grade $3 / 4$ adverse events, $\%$} & \multicolumn{2}{|c|}{ Dinutuximab beta STI } & \multicolumn{2}{|c|}{ Dinutuximab beta LTI } \\
\hline & Alone $\left(n=186^{\mathrm{a}}\right)$ & $+\operatorname{IL}-2\left(n=192^{\mathrm{a}}\right)$ & Alone $\left(n=171^{\mathrm{a}}\right)$ & $\begin{array}{l}+50 \% \\
\text { IL-2 } \\
\left(n=150^{\mathrm{a}}\right)\end{array}$ \\
\hline Immunotherapy-related pain & 16 & 26 & 7 & 18 \\
\hline Fever & 14 & 40 & 13 & 29 \\
\hline Hypersensitivity reaction & 10 & 20 & 3 & 3 \\
\hline Capillary leak syndrome & 4 & 15 & 4 & 8 \\
\hline Diarrhoea & 7 & 21 & 11 & 12 \\
\hline \multicolumn{5}{|l|}{ Neurotoxicity } \\
\hline Central & 2 & 6 & 1 & 1 \\
\hline Peripheral & 1 & 2 & 1 & 1 \\
\hline AST/ALT & 17 & 23 & 21 & 26 \\
\hline \multicolumn{5}{|l|}{ Haematological toxicities } \\
\hline Haemoglobin & 42 & 66 & 53 & 66 \\
\hline White blood cells & 26 & 36 & 32 & 33 \\
\hline Granulocytes & 33 & 58 & 43 & 67 \\
\hline Platelets & 34 & 61 & 33 & 51 \\
\hline Hypotension & 7 & 17 & 2 & 3 \\
\hline Infection & 26 & 34 & 32 & 33 \\
\hline General condition & 16 & 41 & 16 & 21 \\
\hline
\end{tabular}

$A L T$ alanine aminotransferase, $A S T$ aspartate aminotransferase, $I L-2$ interleukin 2, LTI long-term infusion, SIOPEN International Society of Paediatric Oncology European Neuroblastoma, STI short-term infusion

${ }^{a}$ Not all patients were evaluable for each adverse event

${ }^{\mathrm{b}}$ Half the dose administered in the STI study $\left(3 \times 10^{6} \mathrm{IU} / \mathrm{m}^{2} /\right.$ day rather than $6 \times 10^{6} \mathrm{IU} / \mathrm{m}^{2} /$ day $)$ 
practice, this article focuses on the management of AEs associated with dinutuximab beta LTI without IL-2.

\section{Optimising Supportive Care for Children Receiving Dinutuximab Beta}

In the absence of formally recognised guidelines for the management of AEs associated with dinutuximab beta, European experts in immunotherapy for NB were brought together in a virtual meeting to provide practical guidance for the management of common AEs in children receiving dinutuximab beta, based on their clinical experience (key recommendations are summarised in Table 2). The experts agreed that while different treatment regimens/schedules of dinutuximab beta were associated with similar AEs, the intensity and/or frequency of these events observed in clinical practice differs among the different approaches, with an improved toxicity profile associated with the LTI schedule and the omission of IL-2. It was considered important not only to include guidance regarding the management of the most common AEs observed in clinical practice (including pain, fever, hypersensitivity reactions, capillary leak syndrome and diarrhoea) but also AEs that might have a greater impact on the patient and their treatment over the longer term (e.g. neuropathy and visual disturbances).

\subsection{General Principles}

The management of AEs associated with dinutuximab beta may be challenging and may vary from patient to patient, institution to institution and country to country. However, in general, dinutuximab beta-related AEs are considered manageable. It is important to note that beyond the first cycle of treatment, the incidence and/or intensity of some AEs, such as pain, allergies or capillary leak syndrome, generally decreases $[10,12]$.

Some AEs such as fever, hypotension and capillary leak syndrome can be difficult to differentiate from those caused by infection and sepsis and can be diagnostically challenging. The management of these symptoms may also vary substantially according to the underlying cause. Other AEs may be related to NB itself, and should be considered when symptoms do not resolve promptly on discontinuation of dinutuximab beta or in response to adequate supportive care. For example, bone pain or central nervous system symptoms can be observed in children with disease relapse. The group was in consensus that once an AE has occurred in a patient and is considered related to dinutuximab beta treatment, steps should be taken to proactively prevent recurrence during the current cycle and subsequent treatment cycles.

Corticosteroids should be avoided for the duration of dinutuximab beta treatment, from 2 weeks before the first dose in the first cycle to 1 week after the last dose in the last cycle, except for life-threatening conditions. Due to their immunosuppressant activities, corticosteroids are likely to interfere with immunotherapy, although studies to prove such an interaction have not been performed [9]. In addition to emergency events, such as severe allergic reactions, the experts recommend the use of corticosteroids for children with adrenal insufficiency and asthma. In the case of severe or life-threatening symptoms (e.g. anaphylaxis) or autoimmune disease (e.g. transverse myelitis), the infusion should be stopped immediately and dinutuximab beta treatment should be permanently discontinued; corticosteroids and intravenous (i.v.) immunoglobulins should be considered for treatment.

Importantly, most AEs associated with dinutuximab beta occur during drug administration and resolve upon infusion completion [10]. Therefore, temporarily interrupting the infusion to give supportive therapy can slow or prevent the progression of symptoms and does not appear to be detrimental. Once symptoms improve, immunotherapy can be continued, except in cases of severe neurotoxicity. The dinutuximab beta Summary of Product Characteristics provides guidance regarding dose interruption and adjustments based on the severity of certain AEs, such as hypersensitivity reactions and capillary leak syndrome [9]. However, the group strongly recommends that AEs should first be managed with supportive therapy, before dose reductions and interruptions are being considered. It is crucial to train medical staff on how to best manage each of the AEs associated with dinutuximab beta. Clinicians should also keep in mind the importance of educating the parents/carers of patients of what to expect in terms of potential AEs with dinutuximab beta as it will help them support their child through treatment.

\subsection{Pain}

As an on-target effect, pain, including neuropathic pain, is commonly experienced in children treated with dinutuximab beta [10-12, 15]. GD2, the target of dinutuximab beta, is not only expressed on the surface of NB cells but also in normal human tissue in the central and peripheral nervous system [16]. Dinutuximab beta can activate the complement cascade at the GD2-expressing peripheral sensory nerve fibres, resulting in neuropathic pain $[9,17]$.

Pain occurs most commonly in the abdomen, extremities, back, chest or joints (arthralgia) [9]. It generally begins immediately after initiation of the infusion during the first cycle and decreases with subsequent treatment cycles [9, $10,12]$. Rarely, pain may be chronic, disrupt sleep and cause altered sensation, requiring long-term treatment. The administration of dinutuximab beta as LTI and the omission of IL-2 from the treatment regimen resulted in decreased morphine usage [12], potentially permitting home-based 
Table 2 Summary of recommendations for the management of common adverse events associated with dinutuximab beta treatment in patients with high-risk neuroblastoma

\begin{tabular}{|c|c|}
\hline Adverse event & Management recommendation \\
\hline Pain & $\begin{array}{l}\text { Non-opioid analgesics Paracetamol or ibuprofen are recommended for the first cycle(s) of dinutuximab beta treatment. In } \\
\text { painless consecutive cycles, the omission of non-opioids can be considered } \\
\text { Gabapentin Start prior to and continue during dinutuximab beta infusion } \\
\text { Start at } 10 \mathrm{mg} / \mathrm{kg} / \text { day } 3 \text { days prior to infusion, increase to } 2 \times 10 \mathrm{mg} / \mathrm{kg} / \mathrm{day} \text { and } 3 \times 10 \mathrm{mg} / \mathrm{kg} / \mathrm{day} \text { in } 2 \text { consecutive days } \\
\text { before the infusion } \\
\text { Gabapentin } 3 \times 10 \mathrm{mg} / \mathrm{kg} / \text { day can be interrupted between courses or given continuously. It should be tapered off at discon- } \\
\text { tinuation of dinutuximab beta } \\
\text { Morphine: Start prior to and continue during dinutuximab beta infusion. Aim for the lowest dose of morphine required for } \\
\text { the shortest possible time } \\
\text { Prior to dinutuximab beta infusion, start continuous i.v. morphine infusion }(0.02-0.05 \mathrm{mg} / \mathrm{kg} / \mathrm{h}) \text { or administer bolus } \\
\text { (0.05-0.1 mg/kg/h) } 2 \mathrm{~h} \text { before infusion } \\
\text { During dinutuximab beta treatment, continue morphine at } 0.03 \mathrm{mg} / \mathrm{kg} / \mathrm{h} \text { or higher if needed } \\
\text { With daily infusions of dinutuximab beta, morphine infusion can be continued at a decreased rate (e.g. } 0.01 \mathrm{mg} / \mathrm{kg} / \mathrm{h}) \text { for } 4 \\
\text { h after the end of dinutuximab beta infusion } \\
\text { For pain uncontrolled by gabapentin, morphine or non-opioid analgesics, additional i.v. ketamine is recommended. Adjust- } \\
\text { ing the rate and dose of dinutuximab beta infusion should also be considered } \\
\text { Hyoscine butylbromide may be given to manage abdominal pain }\end{array}$ \\
\hline Fever & $\begin{array}{l}\text { Prophylactic antipyretics such as paracetamol or metamizole (where licensed) can be given } \\
\text { Fever can be treated with paracetamol, ibuprofen or metamizole (where licensed) } \\
\text { Blood cultures should be taken to rule out an infection } \\
\text { Febrile neutropenia should be treated with antibiotics according to local guidelines at least until blood cultures return a } \\
\text { negative result and the patient does not present with other features suggestive of active bacterial infection } \\
\text { Fever persisting more than } 48-72 \text { h despite adequate management should prompt ruling out other causes of fever (e.g. } \\
\text { fungus, virus, etc.) as per local guidelines } \\
\text { If fever is not extremely high and is well tolerated, supportive therapy may not be needed }\end{array}$ \\
\hline Hypersensitivity reactions & $\begin{array}{l}\text { i.v. antihistamines (e.g. diphenhydramine) should be administered } \sim 20 \text { min before each dinutuximab beta infusion and } \\
\text { repeated every } 4-6 \mathrm{~h} \text { as required } \\
\text { Oral antihistamines may also be given the day before dinutuximab beta infusion } \\
\text { For grade } 1-2 \text { reactions, dinutuximab beta might be interrupted or the infusion rate reduced and symptoms treated } \\
\text { For grade } \geq 3 \text { reactions, dinutuximab beta should be interrupted immediately and symptoms treated } \\
\text { For severe, life-threatening reactions, the infusion should be stopped immediately and dinutuximab beta treatment should be } \\
\text { permanently discontinued. i.v. antihistamine, adrenaline and steroids should be administered } \\
\text { Intramuscular adrenaline or slow i.v. adrenaline may also be used in patients with anaphylaxis } \\
\text { The use of steroids should be limited to severe and life-threatening reactions } \\
\text { For more detailed guidance, see flow charts in Fig. } 2\end{array}$ \\
\hline Capillary leak syndrome & $\begin{array}{l}\text { Blood pressure and weight should be monitored regularly to guide management } \\
\text { Mild symptoms may require fluid management: } \\
\text { Fluids may be given or restricted dependent on weight/blood pressure and local protocols } \\
\text { Severe symptoms should be managed with fluid restriction } \\
\text { Diuretics should be used with caution } \\
\text { Furosemide may be given to manage severe weight gain with lung oedema or ascites } \\
\text { In severe cases, human albumin solution might be used if albumin levels are low } \\
\text { Some patients may require oxygen support for hypoxaemia } \\
\text { For very rare, severe symptoms, dinutuximab beta infusion may be slowed or interrupted }\end{array}$ \\
\hline Visual disturbances & $\begin{array}{l}\text { Patients with pupillary palsy may temporarily require corrective glasses } \\
\text { Sunglasses are recommended for patients whose symptoms are exacerbated by sunlight } \\
1 \% \text { pilocarpine can be given to improve symptoms } \\
\text { Patients should undergo complete ophthalmological examination and be monitored }\end{array}$ \\
\hline Diarrhoea & $\begin{array}{l}\text { For mild diarrhoea, fluids should be given } \\
\text { For severe diarrhoea, loperamide or racecadotril can be administered }\end{array}$ \\
\hline Neurotoxicity & $\begin{array}{l}\text { For moderate neuropathy, dinutuximab beta should be interrupted and may be resumed once symptoms have resolved } \\
\text { For severe peripheral neuropathy or transverse myelitis, the infusion should be stopped immediately and dinutuximab beta } \\
\text { treatment should be permanently discontinued; i.v. IgG and high-dose steroids should be administered }\end{array}$ \\
\hline Hepatotoxicity & $\begin{array}{l}\text { No management required if no other symptoms present } \\
\text { If transaminase levels are }<20 \text { times normal, patients should be monitored closely } \\
\text { Concomitant hepatotoxic drugs should be avoided }\end{array}$ \\
\hline Haematological toxicities & Generally, no management required other than support with blood products according to local practice \\
\hline Laboratory abnormalities & $\begin{array}{l}\text { Generally, no management required } \\
\text { Ion disturbances should be monitored frequently and fluids should be corrected if needed }\end{array}$ \\
\hline
\end{tabular}

$\operatorname{Ig} G$ immunoglobulin, i.v. intravenous 
administration of dinutuximab beta. Pain was generally considered well managed by pain relief, with infrequent grade $3 / 4$ pain when appropriate pain relief measures were given.

\subsubsection{Management}

Pain should be treated aggressively by using triple therapy (non-opioid analgesics, gabapentin and morphine). Nonopioid analgesics should be given throughout treatment, e.g. paracetamol or ibuprofen. The experts also recommend the use of metamizole, where licensed for use, and highlight that the type of non-opioid analgesic used depends on the tolerability of the individual patient and the preference of the individual institution.

The use of gabapentin before and during dinutuximab beta infusion is strongly recommended. Gabapentin has to be gradually increased to ensure that the patient is on the full dose regimen prior to dinutuximab beta infusion [9]. A dose of $10 \mathrm{mg} / \mathrm{kg} /$ day should be administered 3 days prior to infusion, which is then increased to $2 \times 10 \mathrm{mg} / \mathrm{kg} /$ day the next day and to $3 \times 10 \mathrm{mg} / \mathrm{kg} / \mathrm{day}$ the day before the infusion to completion of the cycle. It should be tapered off and discontinued between cycles and after completion of dinutuximab beta infusion. Alternatively, it can be continued between cycles at full dose or tapered to $10 \mathrm{mg} / \mathrm{kg} / \mathrm{day}$, especially in patients with lasting pain.

The use of morphine has also been recommended as premedication and during dinutuximab beta treatment. The first infusion day and cycle usually require higher doses than subsequent days and cycles. However, morphine schedules vary among different hospitals and different countries. The experts agreed that a continuous i.v. morphine infusion $(0.02-0.05 \mathrm{mg} / \mathrm{kg} / \mathrm{h})$ should be started prior to dinutuximab beta treatment or a bolus $(0.05-0.1 \mathrm{mg} / \mathrm{kg})$ should be administered $2 \mathrm{~h}$ before antibody infusion. Subsequently, a continuous morphine infusion dosing rate of $0.03 \mathrm{mg} / \mathrm{kg} / \mathrm{h}$ (or higher if needed) is recommended concomitantly with dinutuximab beta infusion, and can be weaned off during dinutuximab beta treatment in the absence of pain. Morphine should be weaned off over a maximum of 5 days by progressively decreasing the dosing rate, with the aim of completing morphine withdrawal by the end of dinutuximab beta infusion in the first cycle, and within 3-5 days from the start of the second cycle. However, some patients may need pain relief medication after completion of dinutuximab infusion.

The group's recommendation was to aim for the lowest dose of morphine required for the shortest possible time to control a patient's pain. Some patients may not require morphine beyond cycle 2 of dinutuximab beta treatment. For pain uncontrolled by gabapentin and morphine, i.v. ketamine may be used in addition to these drugs. However, fewer patients require ketamine when dinutuximab beta is administered using the LTI schedule rather than the STI schedule. Hyoscine butylbromide may be given to manage abdominal pain. For uncontrolled pain, adjusting the rate and dose of the dinutuximab beta infusion should also be considered.

\subsection{Fever}

Fever, which is considered to be an axillary temperature of $\geq 38^{\circ} \mathrm{C}$, is a common $\mathrm{AE}$ associated with dinutuximab beta treatment [11]. Occasionally, patients receiving dinutuximab beta experience temperatures of $40{ }^{\circ} \mathrm{C}$ or higher. Although fever related to dinutuximab beta can occur at any time during and shortly after dinutuximab beta infusion, it is generally observed during the first few days of infusion. Fever that is experienced later during treatment may also be due to an infection, indicating the need for a differential diagnosis. Because the introduction of the LTI schedule and the omission of IL-2 from the treatment regimen has been associated with a lower frequency of dinutuximab-related fever in clinical practice, infections should be considered earlier during treatment. Given that fever is experienced by many patients and is often a cause for concern, educating the patient, family and staff about fever prior to beginning dinutuximab beta therapy may be helpful.

\subsubsection{Management}

As fever is an expected AE of immunotherapy, prophylactic antipyretics, such as paracetamol, can be given, particularly in patients who did not tolerate fever well in previous treatment cycles. Where licensed for use, intermittent or continuous infusion of metamizole may also be administered to prevent fever.

If a patient develops a fever, blood cultures should be taken to rule out an infection, which, if confirmed, should be treated appropriately (i.e. with antibiotics), particularly in the event of concomitant neutropenia. Generally, empiric antibiotics are not recommended in patients with fever alone, even in the case of mild C-reactive protein elevation, unless there is neutropenia or clinical suspicion of infection.

Febrile patients can generally be treated with paracetamol. If children do not respond well to paracetamol, ibuprofen may be given as an alternative with appropriate gastroprotectants (e.g. proton-pump inhibitors). According to some experts, ibuprofen administered as half dose every $4 \mathrm{~h}$ as opposed to full dose every $8 \mathrm{~h}$ may also be effective. In addition, metamizole was considered a good alternative for fever relief, but it is not available in all EU countries. Once antipyretics are initiated to treat fever in a patient, consideration should be given to administering regular antipyretics during the next two subsequent dinutuximab beta cycles. 
The experts agreed that, if the fever is stable, well tolerated by patients and not extremely high, supportive therapy may not be needed. Fans and cold packs can be used if the patient finds them helpful.

\subsection{Hypersensitivity Reactions}

Due to the immunotoxic effects of monoclonal antibodies [18], hypersensitivity reactions are an expected AE in patients receiving dinutuximab beta $[10,11,15]$. Hypersensitivity reactions may include mild symptoms (e.g. localised cutaneous reactions), moderate symptoms (e.g. hypotension), or severe symptoms (e.g. bronchospasm, angioedema or anaphylactic shock) [10]. While dinutuximab beta has previously been associated with severe reactions, the use of premedication, LTI and the omission of IL-2 has resulted in most hypersensitivity reactions now being mild and cutaneous (rash/pruritus). Severe reactions, such as anaphylaxis, are rare. If they do occur, anaphylactic reactions can be experienced as early as within a few minutes of the first dinutuximab beta infusion and are commonly associated with bronchospasm and urticaria [9].

\subsubsection{Management}

As hypersensitivity reactions are expected in association with immunotherapy, premedication is recommended to prevent such reactions. The consensus was that i.v. antihistamines (e.g. diphenhydramine, chlorphenamine) should be administered $\sim 20$ min before starting each dinutuximab beta infusion and repeated every $4-6 \mathrm{~h}$ as required. Alternatively, oral antihistamines may be given regularly from the day before starting the dinutuximab beta infusion. Patients should be closely monitored for anaphylaxis and allergic reactions, particularly during the first and second treatment cycle.

Mild symptoms may resolve quickly, but in rare cases may develop into more serious symptoms, such as hypotension or bronchospasm. For mild/moderate hypersensitivity reactions (grade 1-2), it is recommended to interrupt dinutuximab beta infusion and administer supportive measures. Once symptoms resolve, the infusion can be resumed. Depending on the symptoms, the starting rate of the infusion could be the original (e.g. for a rash) or reduced and then gradually increased (e.g. for bronchospasm or angioedema). In the case of grade $\geq 3$ hypersensitivity reactions, dinutuximab beta infusion should be interrupted immediately and symptoms should be treated appropriately. Treatment may be resumed for subsequent cycles. For severe, lifethreatening reactions (grade 3-4 anaphylaxis), the infusion should be stopped immediately and dinutuximab beta treatment should be permanently discontinued; i.v. antihistamine, adrenaline (i.v. bolus every 3-5 $\mathrm{min}$ ) and steroids, such as methylprednisolone (i.v. bolus) or hydrocortisone, should be administered, according to clinical response. The group also recommends the use of intramuscular adrenaline or slow i.v. adrenaline in patients with anaphylaxis. Antihistamines commonly used to manage hypersensitivity reactions include cetirizine, chlorphenamine, diphenhydramine, clemastine, dexchlorpheniramine and levocetirizine. For bronchial and/ or pulmonary hypersensitivity reactions, local guidelines may recommend the use of salbutamol, corticosteroids and/ or inhalation with adrenaline every $2 \mathrm{~h}$. Levodropropizine or an adrenaline nebuliser can be used to treat cough. More guidance on the management of specific hypersensitivity reactions is provided in Figs. 2 and 3.

\subsection{Capillary Leak Syndrome}

Capillary leak syndrome may develop within hours after initiation of dinutuximab beta treatment and/or IL-2 [9]. It can present with a variety of symptoms, including weight gain, fever, fatigue, light headedness, headache, rash, breathing difficulties, hypotension and tachycardia [9]. Whereas severe symptoms are rarely observed in schedules without IL-2, the LTI administration may also reduce capillary leak syndrome as compared to the STI schedule of dinutuximab beta. Mild symptoms such as puffiness and increased weight are still common with LTI. Similar to other AEs, such as pain, the frequency of capillary leak syndrome is highest during the first treatment cycle and decreases with subsequent cycles [10].

\subsubsection{Management}

The management of capillary leak syndrome varies among institutions. In general, the patient's blood pressure should be closely monitored along with their weight to guide the management of symptoms. The experts agreed that mild capillary leak syndrome does not always require treatment; however, when it does, fluid management is indicated. Fluids may either be administered or restricted dependent on the patient's weight/blood pressure and local protocols. Whereas in some institutions capillary leak syndrome is routinely managed with fluid restriction, in others, the usage is limited to severe cases. Due to the risk of decreased arterial pressure, diuretics should be used with caution. Generally, albumin infusions are not required, as albumin levels return to normal over time. However, some hospitals may use long-term albumin infusion in addition to diuretics in the case of severe fluid retention. The group also recommends the use of furosemide to manage severe symptoms, such as severe weight gain with lung oedema or ascites if the arterial pressure is normal. Some patients may also require oxygen 
a

Allergic reaction: Rash

Examine the patient and make sure no bronchospasm or angioedema is present

Start regular antihistamine (e.g. diphenhydramine) and continue antibody infusion at the same rate

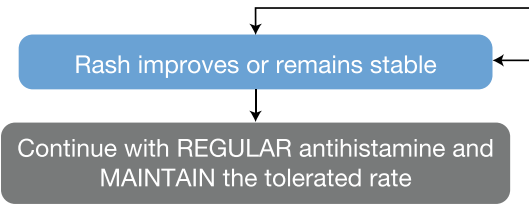

b

Allergic reaction: Angioedema

Stop (DO NOT disconnect) antibody infusion

Give antihistamine (e.g. diphenhydramine)

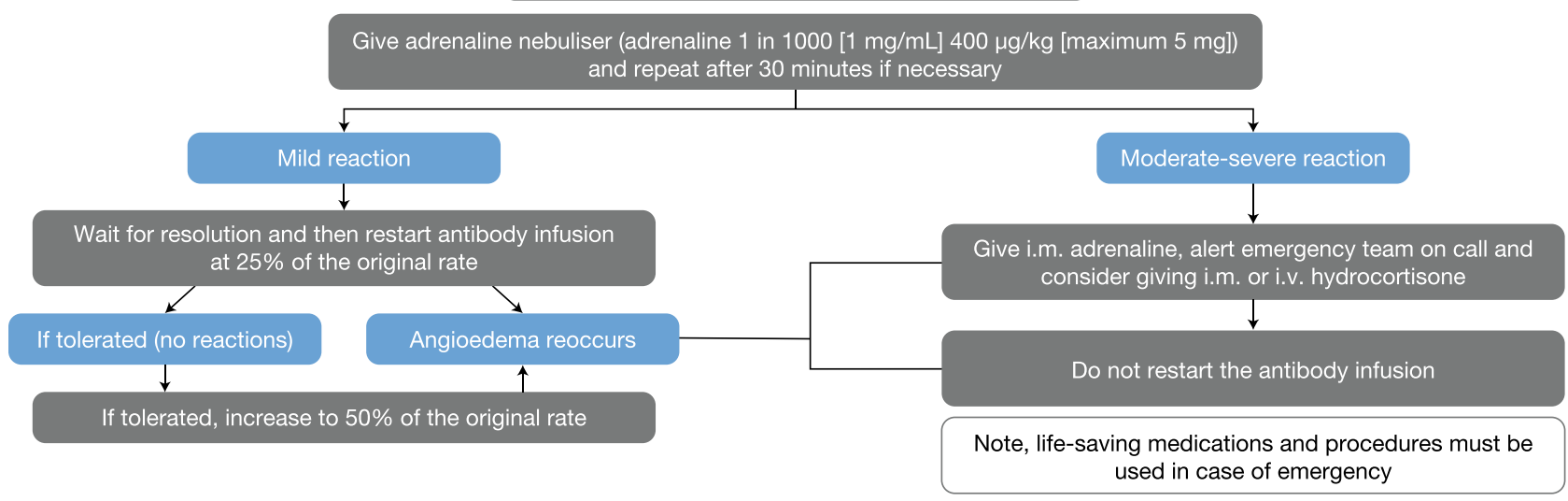

Fig. 2 Guidance on the management of $\mathbf{a}$ rash and $\mathbf{b}$ angioedema. i.m. intramuscular, i.v. intravenous

support for hypoxaemia, particularly at night. For very rare, severe cases that do not respond to therapy or require vasoactive amines or ventilatory support, consideration should be given to slowing or interrupting dinutuximab beta infusion.

\subsection{Visual Disturbances}

There is very little information in the literature regarding visual disturbances in association with dinutuximab beta therapy [19], and this expert group believes that these events are under-reported in clinical studies. Ocular abnormalities, such as mydriasis and impaired accommodation, have also been reported with the humanised antiGD2 antibody hu14.18K322A [20]. An unusual dilation or widening of the pupils, often referred to as pupillary palsy, mydriasis and iridoplegia, is frequently experienced in patients receiving dinutuximab beta, particularly in the later stages of treatment. It can occur without visual problems or with mild and transient visual disturbances, and can be difficult to diagnose, as children often do not 
a

\section{Allergic reaction: Bronchospasm}

Stop (DO NOT disconnect) antibody infusion

Give salbutamol nebuliser and diphenhydramine

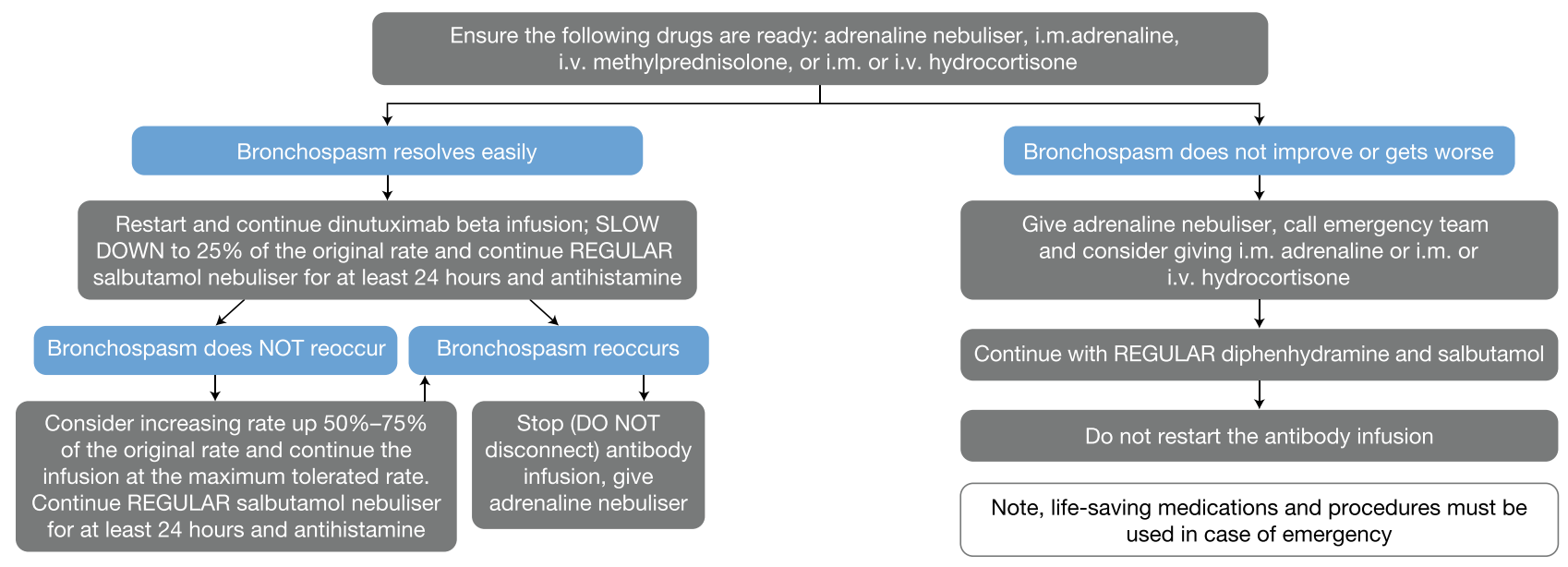

b

Allergic reaction: Anaphylaxis

Stop and DISCONNECT antibody infusion

Give i.m. adrenaline

Inform the emergency team on site

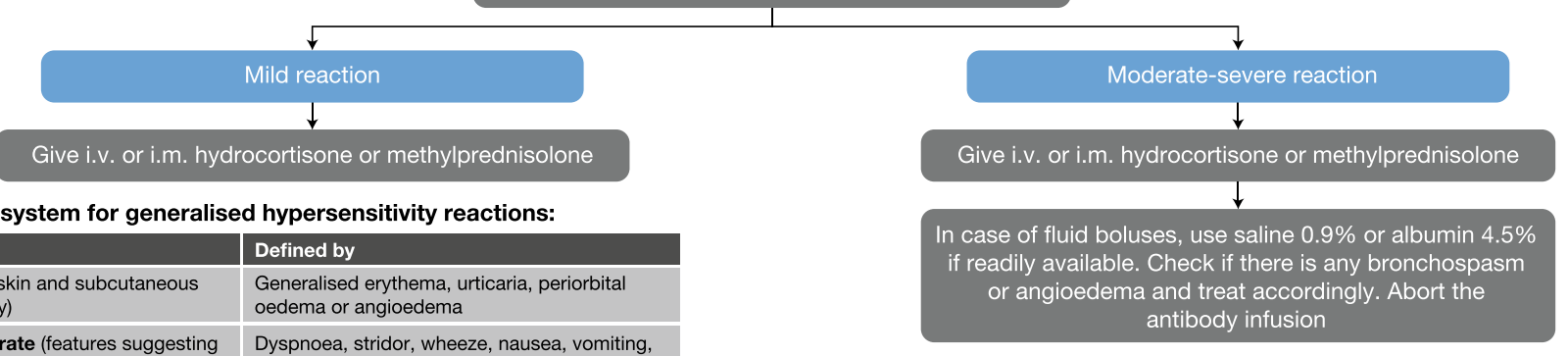

Dyspnoea, stridor, wheeze, nausea, vomiting, dizziness (presyncope), diaphoresis, chest or throat tightness or abdominal pain

respiratory, cardiovascular or gastrointestinal involvement)

Cyanosis or $\mathrm{SpO} 2 \leq 92 \%$ at any stage, hypotension (SBP $<90 \mathrm{~mm} \mathrm{Hg}$ in adults), confusion, collapse, LOC or incontinence

Note, life-saving medications and procedures must be used in case of emergency. Treatment with dinutuximab beta should be permanently discontinued if grade 3 or 3 - Severe (hypoxia, hypotension or neurologic compromise) 4 anaphylaxis occurs

Fig. 3 Guidance on the management of a bronchospasm and $\mathbf{b}$ anaphylaxis. i.m. intramuscular, i.v. intravenous, $L O C$ loss of consciousness, $S B P$ systolic blood pressure, $\mathrm{SpO}_{2}$ oxygen saturation

complain of symptoms. Pupillary palsy might resolve after several months following the completion of dinutuximab beta treatment, but sometimes, may persist for years.

\subsubsection{Management}

The consensus of the group was that patients with pupillary palsy may (temporarily) require corrective glasses. For patients whose symptoms are exacerbated by sunlight, sunglasses are recommended. In addition, symptoms may be improved with the use of $1 \%$ pilocarpine. If there are no additional neurological effects, no dose adjustment or interruption of dinutuximab beta therapy is required. The experts recommend complete ophthalmological examination and regular monitoring of patients. 


\subsection{Diarrhoea}

Diarrhoea is a common AE associated with dinutuximab beta in clinical practice, often presenting during later stages of infusion and/or after discharge from hospital. It is believed to be related to capillary leak syndrome and the discontinuation of morphine.

\subsubsection{Management}

According to the experts, patients with mild diarrhoea may only require fluid support, whereas those with severe diarrhoea can be given loperamide. Racecadotril is an alternative agent recommended for the treatment of diarrhoea.

\subsection{Neurotoxicity}

Central neurotoxicity, such as transverse myelitis, has also been reported in patients receiving dinutuximab beta. Although the incidence of this type of neurotoxicity has been reported to be lower in the absence of IL-2, it can still occur when dinutuximab beta is given without IL-2 [11].

\subsubsection{Management}

In the case of transverse myelitis, the infusion should be stopped immediately and dinutuximab beta treatment should be permanently discontinued; i.v. immunoglobulin and highdose steroids should be administered. A few patients with such neuropathy have also been successfully treated with plasmapheresis, but the benefits are not fully understood. The group strongly agreed that children experiencing severe neuropathy should not be rechallenged with dinutuximab beta even after complete recovery.

\subsection{Hepatotoxicity}

Increased levels of liver transaminases have also been reported with dinutuximab beta treatment $[10,11]$, especially in patients who received busulfan/melphalan or experienced veno-occlusive disease prior to dinutuximab beta. Generally, these events are not severe; they are transient and are considered related to concomitant hepatotoxic medications.

\subsubsection{Management}

The consensus was that hepatotoxicity does not require management if no other symptoms are present. If transaminase levels are $<20$ times the upper normal limit, the patient can be monitored closely without reducing or interrupting dinutuximab beta. Hepatotoxic drugs should be avoided.

\subsection{Haematological Toxicities}

Low-grade haematological toxicities, including thrombocytopaenia, leucopenia and anaemia, are often observed in patients who have undergone stem cell transplant, and are usually self-limiting.

\subsubsection{Management}

In general, haematological toxicities resolve with time and do not require specific management or modifications of dinutuximab beta administration.

\subsection{Laboratory Abnormalities}

Laboratory abnormalities, such as high C-reactive protein levels, low albumin levels or ion disturbances, may also occur in patients receiving dinutuximab beta treatment.

\subsubsection{Management}

In general, laboratory abnormalities resolve with time and do not require specific management or modifications of dinutuximab beta administration. Ion disturbances should be monitored frequently and fluids should be corrected if needed.

\section{Conclusions}

Dinutuximab beta immunotherapy is recommended by SIOPEN as part of the standard of care for patients with high-risk NB [11]. However, dinutuximab beta has been associated with various AEs, which may be a challenge for clinicians who may not be experienced in managing immunotherapy-related toxicities, such as severe pain, persistent fever, hypersensitivity reactions and capillary leak syndrome. Clinicians should also be aware of the potential long-term effects of dinutuximab beta treatment, including visual disturbances, peripheral neuropathy and transverse myelitis, and how to manage these. This practical guidance for the management of AEs in patients receiving dinutuximab beta will facilitate their treatment, allowing them to receive the intended treatment doses of dinutuximab beta to gain the maximum benefit with minimal toxicity.

Acknowledgements Editorial assistance was provided by Katrin Male of $\mathrm{mXm}$ Medical Communications funded by EUSA Pharma. 


\section{Declarations}

Funding The virtual advisory board meeting that led to the development of this article and editorial assistance for this article were funded by EUSA Pharma.

Conflict of interest GB, FB, BB, ASD, FH, CM, DR, PMR, AW and $\mathrm{MvN}$ received compensation for their role as a consultant/advisor at the EUSA Pharma Advisory Board meeting that has led to the subsequent development of this publication. GB has also received educational funding from EUSA Pharma. AB declares that she has no conflicts of interest that might be relevant to the contents of this article. FB had a consultant or advisory role and support for attending symposia from EUSA Pharma. ASD has received support for attending symposia from EUSA Pharma. FH has received support for attending symposia from EUSA Pharma. DR received support for attending symposia from EUSA Pharma. PMR had a consultant or advisory role and support for attending symposia from EUSA Pharma. AW has received support for attending symposia from EUSA Pharma and has a consulting agreement with EUSA Pharma.

Ethics approval Not applicable.

Consent to participate Not applicable.

Consent for publication Not applicable.

Availability of data and material Not applicable.

Code availability Not applicable.

Authors' contributions All authors were involved in the development, review and approval of the manuscript.

Open Access This article is licensed under a Creative Commons Attribution-NonCommercial 4.0 International License, which permits any non-commercial use, sharing, adaptation, distribution and reproduction in any medium or format, as long as you give appropriate credit to the original author(s) and the source, provide a link to the Creative Commons licence, and indicate if changes were made. The images or other third party material in this article are included in the article's Creative Commons licence, unless indicated otherwise in a credit line to the material. If material is not included in the article's Creative Commons licence and your intended use is not permitted by statutory regulation or exceeds the permitted use, you will need to obtain permission directly from the copyright holder. To view a copy of this licence, visit http://creativecommons.org/licenses/by-nc/4.0/.

\section{References}

1. Swift CC, Eklund MJ, Kraveka JM, Alazraki AL. Updates in diagnosis, management, and treatment of neuroblastoma. Radiographics. 2018;38:566-80. https://doi.org/10.1148/rg.2018170132

2. Steliarova-Foucher E, Colombet M, Ries LAG, Moreno F, Dolya A, Bray F, et al. International incidence of childhood cancer, 2001-10: a population-based registry study. Lancet Oncol. 2017;18:719-31. https://doi.org/10.1016/S1470-2045(17)30186-9

3. Pinto NR, Applebaum MA, Volchenboum SL, Matthay KK, London WB, Ambros PF, et al. Advances in risk classification and treatment strategies for neuroblastoma. J Clin Oncol. 2015;33:3008-17. https://doi.org/10.1200/JCO.2014.59.4648
4. Whittle SB, Smith V, Dohert E, Zhao S, McCarty S, Zage PE. Overview and recent advances in the treatment of neuroblastoma. Expert Rev Anticancer Ther. 2017;17:369-86. https://doi.org/10. 1080/14737140.2017.1285230

5. Smith V, Foster J. High-risk neuroblastoma treatment review. Children. 2018;5:1-7. https://doi.org/10.3390/children5090114

6. Yu AL, Gilman AL, Ozkaynak MF, London WB, Kreissman SG, Chen HX, et al. Anti-GD2 antibody with GM-CSF, interleukin-2, and isotretinoin for neuroblastoma. N Engl J Med. 2010;363:1324-34. https://doi.org/10.1056/NEJMoa0911123

7. Yu AL, Gilman AL, Ozkaynak MF, Naranjo A, Diccianni MB, Gan J, et al. Long-term follow-up of a phase III study of ch14.18 (Dinutuximab) + cytokine immunotherapy in children with high-risk neuroblastoma: COG Study ANBL0032. Clin Cancer Res. 2021;27:2179-89. https://doi.org/10.1158/1078-0432. CCR-20-3909

8. Unituxin (dinutuximab) Prescribing Information. 2015. https:// www.accessdata.fda.gov/drugsatfda_docs/label/2015/125516s000 lbl.pdf. Accessed Feb 2021

9. Qarziba (dinutuximab beta) Summary of Product Characteristics (28/01/2020). https://www.ema.europa.eu/en/documents/productinformation/qarziba-epar-product-information_en.pdf. Accessed Dec 2020

10. Ladenstein RL, Poetschger U, Valteau-Couanet D, Luksch R, Castel V, Yaniv I, et al. Interleukin 2 with anti-GD2 antibody ch14.18/ $\mathrm{CHO}$ (dinutuximab beta) in patients with high-risk neuroblastoma (HR-NBL1/SIOPEN): a multicentre, randomised, phase 3 trial. Lancet Oncol. 2018;19:1617-29. https://doi.org/10.1016/S14702045(18)30578-3

11. Ladenstein RL, Poetschger U, Valteau-Couanet D, Gray J, Luksch $\mathrm{R}$, Balwierz W, et al. Randomization of dose-reduced subcutaneous interleukin-2 (scIL2) in maintenance immunotherapy (IT) with anti-GD2 antibody dinutuximab beta (DB) long-term infusion (LTI) in front-line high-risk neuroblastoma patients: early results from the HR-NBL1/SIOPEN trial. J Clin Oncol. 2019;37:Abstr 10013. https://doi.org/10.1200/JCO.2019.37.15_ suppl.10013

12. Mueller I, Ehlert K, Endres S, Pill L, Siebert N, Kietz S, et al. Tolerability, response and outcome of high-risk neuroblastoma patients treated with long-term infusion of anti-GD2 antibody ch14.18/CHO. MAbs. 2018;10:55-61. https://doi.org/10.1080/ 19420862.2017.1402997

13. Sait S, Modak S. Anti-GD2 immunotherapy for neuroblastoma. Expert Rev Anticancer Ther. 2017;17:889-904. https://doi.org/ 10.1080/14737140.2017.1364995

14. Ladenstein RL, Poetschger U, Pearson ADJ, Brock P, Luksch R, Castel V, et al. Busulfan and melphalan versus carboplatin, etoposide, and melphalan as high-dose chemotherapy for high-risk neuroblastoma (HR-NBL1/SIOPEN): an international, randomised, multi-arm, open-label, phase 3 trial. Lancet Oncol. 2017;18:50014. https://doi.org/10.1016/S1470-2045(17)30070-0

15. Lode H, Valteau-Couanet D, Gray J, Luksch R, Wieczorek A, Castel V, et al. Randomised use of Anti-GD2 antibody dinutuximab beta (DB) long-term infusion with and without subcutaneous interleukin-2 (scIL-2) in high-risk neuroblastoma patients with relapsed and refractory disease: results from the SIOPEN LTItrial. J Clin Oncol. 2019;37:Abstr 10014. https://doi.org/10.1200/ JCO.2019.37.15_suppl.10014

16. Lammie G, Cheung N, Gerald W, Rosenblum M, Cordoncardo C. Ganglioside GD(2) expression in the human nervous-system and in neuroblastomas: an immunohistochemical study. Int J Oncol. 1993;3:909-15. https://doi.org/10.3892/ijo.3.5.909

17. Keyel ME, Reynolds CP. Spotlight on dinutuximab in the treatment of high-risk neuroblastoma: development and place in therapy. Biologics. 2018;13:1-12. https://doi.org/10.2147/BTT.S1145 30 
18. Brennan FR, Morton LD, Spindeldreher S, Kiessling A, Allenspach R, Hey A, et al. Safety and immunotoxicity assessment of immunomodulatory monoclonal antibodies. MAbs. 2010;2:23355. https://doi.org/10.4161/mabs.2.3.11782

19. Blom T, Lurvink R, Aleven L, Mensink M, Wolfs T, Dierselhuis $\mathrm{M}$, et al. Treatment-related toxicities during anti-GD2 immunotherapy in high-risk neuroblastoma patients. Front Oncol. 2021;10: 601076. https://doi.org/10.3389/fonc.2020.601076

20. Tse BC, Navid F, Billups CA, O’Donnell T, Hoehn ME. Ocular abnormalities in patients treated with a novel anti-GD2 monoclonal antibody, hu14.18K322A. J AAPOS. 2015;19:112-5. https:// doi.org/10.1016/j.jaapos.2014.11.005

\section{Authors and Affiliations}

\section{Giuseppe Barone ${ }^{1} \cdot$ Ailish Barry $^{1} \cdot$ Francisco Bautista $^{2} \cdot$ Bénédicte Brichard $^{3}$. Anne-Sophie Defachelles ${ }^{4}$.} Fiona $\mathrm{Herd}^{5}$. Carla Manzitti ${ }^{6}$. Dirk Reinhardt ${ }^{7} \cdot$ Pedro M. Rubio $^{8} \cdot$ Aleksandra Wieczorek $^{9} \cdot$ Max M. $^{\text {Man Noesel }}{ }^{10,11}$

1 Department of Paediatric Oncology, Great Ormond Street Hospital for Children, London, UK

2 Department of Paediatric Haematology and Oncology, Hospital Universitario Niño Jesus, Madrid, Spain

3 Cliniques Universitaires Saint-Luc, Université Catholique de Louvain, Brussels, Belgium

4 Pediatric and AYA Oncology Unit, Centre Oscar-Lambret, Lille Cedex, France

5 Department of Paediatric Oncology, Royal Aberdeen Children's Hospital, Aberdeen, UK

6 Oncology Unit, Istituto Giannina Gaslini, Genova, Italy

7 Pediatrics III, Pediatric Hematology/Oncology, University Hospital Essen, Essen, Germany
8 Pediatric Hemato-Oncology Department, Hospital Universitario La Paz, Madrid, Spain

$9 \quad$ Pediatric Oncology-Hematology Department, Institute of Pediatrics, Jagiellonian University Medical College, Kraków, Poland

10 Department of Solid Tumors, Princess Máxima Center for Pediatric Oncology, Heidelberglaan 25, 3584 CS Utrecht, The Netherlands

11 Division Cancer and Imaging, University Medical Center Utrecht, Utrecht, The Netherlands 